\title{
A Circulated Block Transmission Scheme for FTN Signaling
}

\author{
Mingqi Li', Shihao Lai ${ }^{2,3}$, Yaqiu Peng² \\ ${ }^{1}$ Wireless Communication Research Center for New Media, Shanghai Advanced Research Institute, CAS, Shanghai, China \\ ${ }^{2}$ University of Chinese Academy of Sciences, Beijing, China \\ ${ }^{3}$ School of Information Science \& Technology, Shanghai Tech University, Shanghai, China \\ Email: limq@sari.ac.cn, laishh@shanghaitech.edu.cn,pengyq@sari.ac.cn
}

How to cite this paper: Li, M.Q., Lai, S.H. and Peng, Y.Q. (2017) A Circulated Block Transmission Scheme for FTN Signaling. Int. J. Communications, Network and System Sciences, 10, 269-279.

https://doi.org/10.4236/ijens.2017.108B029

Received: July 4, 2017

Accepted: August 11, 2017

Published: August 14, 2017

\begin{abstract}
Fast-Than-Nyquist (FTN) transmission is a promising method to improve the spectrum efficiency for future wireless communication systems. However, this benefit of FTN is at the price of inducing the inter-symbol interference (ISI), which increases the complexity of the receiver. In this paper, a circulated block transmission scheme for FTN signaling, i.e. CB-FTN system is proposed. The detail implementation structure of CB-FTN transceiver is presented, in which the ISI caused by FTN transmission is canceled by the frequency-domain equalization (FDE), and the inter-block interference (IBI) caused by the multi-path channel is overcome by the cyclic-prefix. The postprocessing signal to noise ratio (pSNR) is analyzed for the CB-FTN receiver with zero-forcing FDE in AWGN channel, which is verified by the simulation results. Moreover, the BER performances and computational complexity of CB-FTN system are compared with the existed scheme.
\end{abstract}

\section{Keywords}

FTN, ISI, Frequency-Domain Equalization, Post-Processing SNR

\section{Introduction}

How to further improve the spectrum efficiency of transmission scheme is one of major issues for future wireless communication systems. Non-orthogonal transmission technologies have opened a door for the breakthrough in the above challenge. One of potential solutions is faster-than-Nyquist (FTN) signaling, which was first studied by Mazo in 1975. It was shown that by intentionally introducing inter-symbol inference (ISI), FTN signaling with sinc pulse can achieve 25\% higher symbol rate than Nyquist signaling in additive white Gaussian noise (AWGN) channels [1]. The investigations in [2] show that the more 
practical root raised cosine (RRC) pulse, rather than the sinc shaping pulses, can also apply in FTN signaling. In [3] a discrete time form of FTN signaling is introduced, which enables same bite-error-rate (BER) performance as continuous FTN signaling with much lower cost computation.

Although the FTN signaling can improve the spectrum efficiency, the inevitable inter-symbol interference (ISI) has to be properly addressed to guarantee the error performance. Therefore, the major concern in FTN signaling is the detection algorithms [4] [5]. Since frequency domain equalization (FDE) is a computation-efficient method to mitigate the ISI, it is also widely applied to FTN systems [6] [7].

Except the equalizer designed for the FTN receiver, some efforts are also put into designing the FTN transmission schemes [8] [9]. In order to remove the effects of the ISI, a cyclic prefix (CP) based FTN block transmission scheme is addressed in [8], which will produce a loss of transmission efficiency. In [9], a CP and cyclic suffix (CS)-assisted symbol block transmission for FTN signaling is presented to overcome the above shortage. However, the redundant symbolbased CP/CS inserting and sample-based CP/CS discarding operations are needed before and after pulse-shaping filtering respectively, so as to avoid increasing transmission overhead to deal with the ISI. Nevertheless, such processing will bring about additional computational complexity at the transmitter.

In [10] and [11], a sample-based circular block transmission scheme is proposed for the filter-bank based wireless communication systems. Due to the specially designed circulated-sample-blocking operation performed after pulseshaping filtering, the outputs of pulse-filtering are circular sample blocks. Therefore, the CP padding, utilized to cancel the inter-block interference (IBI) caused by multi-path channel, will not bring about the additional out-of-band leakage, which, otherwise, will be induced by the discontinuity between the $\mathrm{CP}$ and sample block.

In this paper, we proposed a circulated block transmission scheme for FTN signaling, i.e. CB-FTN system. By circulated sample blocking and CP padding, the CB-FTN system can achieve higher transmission rate than that of Nyquist system and meanwhile eliminate the IBI by FDE with the estimated channel frequency response. In addition, by exploiting the circulation property of the sample block, the ISI can be canceled by FDE with the circular self-correlation function of pulse-shaping filter as well.

\section{Sampling of FTN Signaling}

The continuous-time FTN signaling can be expressed as

$$
s(t)=\sum_{d=-\infty}^{\infty} a(d) p(t-d \xi T)
$$

where $a(d)$ is the input constellation symbols, $p(t)$ is the impulse response of $T$-orthogonal Nyquist pulse-shaping filter with unit energy. $\xi$ is the time squeezing factor, and $\xi<1$. 
Assume $\xi T$ is integer times of the sampling interval $\Delta T$, i.e. $\xi T=N_{F T N} \Delta T$, $N_{\text {FTN }}$ is a integer. Then, the sampled discrete-time FTN signaling can be obtained as

$$
s(n)=\sum_{d=-\infty}^{\infty} a(d) p\left(n-d N_{F T N}\right)
$$

By properly system parameters designing, $T$ can also be set to be integer times of $\Delta T$, i.e. $T=N_{S} \times \Delta T$, where $N_{S}$ can be viewed as the up-sampling rate of $p(t)$. Therefore, $p(n)$ is a $\mathrm{N}$-orthogonal discrete Nyquist pulse-shaping filter, and satisfies the orthogonal condition

$$
\sum_{n=0}^{L-1} p(n) p^{*}\left(n-d N_{S}\right)= \begin{cases}1, & d=0 \\ 0, & d \neq 0\end{cases}
$$

where $L$ is the length of $p(n)$. In fact, if $N_{F T N}=N_{S}$, i.e. $\xi=1$, (1) and (2) become Nyquist transmission.

\section{CB-FTN System Model}

\subsection{Structure of CB-FTN Transmitter}

The structure of the CBT-FTN transmitter is illustrated in Figure 1. We suppose that the input constellation symbol sequence is segmented, and, w.l.o.g., one of symbol segments, i.e. one data symbol block, can be expressed as $a(d)$ for $0 \leq d \leq D-1$. Note that $d$ and $D$ are the index and the number of symbols transmitted during each symbol block, respectively.

After the FTN filtering, the output signal could be expressed as

$$
f(n)=\sum_{d=0}^{D-1} a(d) p\left(n-d N_{F T N}\right)
$$

Then, by circulated blocking processing, the output signal could be given by

$$
b(n)=\sum_{d=0}^{D-1} a(d) p\left(\left(n-d N_{F T N}\right)\right)_{Q}, \quad 0 \leq n \leq Q-1
$$

where $Q=D \times N_{F T N}$ and $Q \geq L,((\cdot))_{Q}$ denotes the Modulo-Q operation.

Finally, the data block is padded with $\mathrm{CP}$ to form the circularly sampleblocked FTN signaling $s(n)$.

\subsection{Structure of CB-FTN Receiver}

The structure of the CB-FTN receiver is illustrated as in Figure 2. Assume the

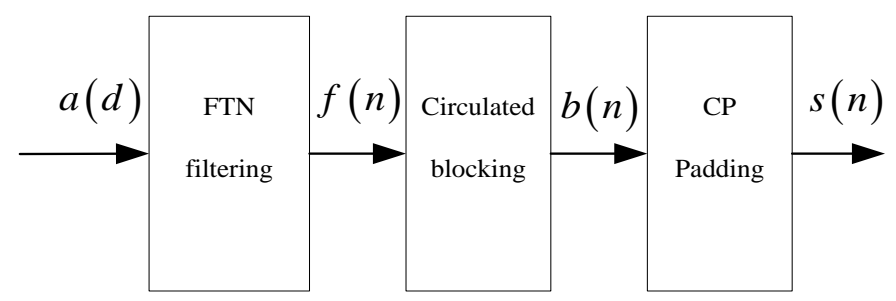

Figure 1. Structure of CB-FTN transmitter. 


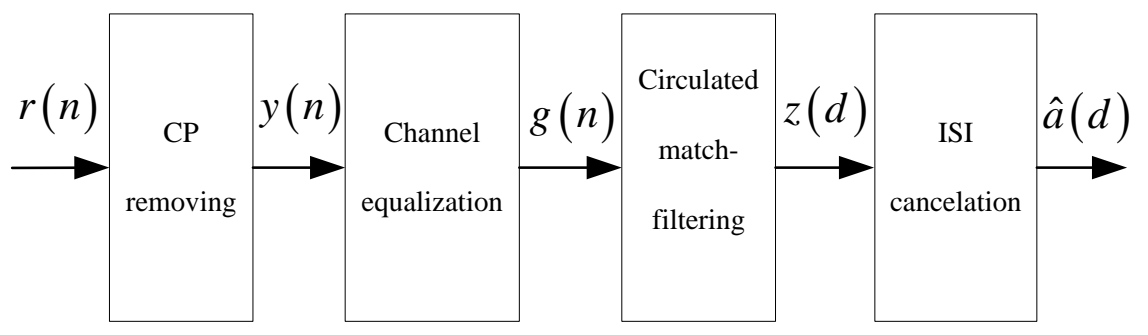

Figure 2. Structure of CB-FTN receiver.

circularly blocked FTN signaling $s(n)$ is passed through the wireless channel, which does not change during one sample block period, and perfect frequency and timing synchronization are achieved at the receiver.

After the CP removing from the received signal $r(n)$, the output is given as

$$
y(n)=b(n) \otimes h(n)+w(n), \quad 0 \leq n \leq Q-1
$$

where $h(n)$ is the channel impulse response with power delay profile less than the CP length, $w(n)$ is the complex-valued AWGN with variance $\sigma^{2}$, and $\otimes$ denotes the cyclic convolution.

Assume the perfect channel estimation is achieved and zero-forcing (ZF) equalization is applied, the output of channel equalization could be expressed as

$$
g(n)=y(n) \otimes \bar{h}(n)=b(n) \otimes h(n) \otimes \bar{h}(n)+w(n) \otimes \bar{h}(n)=b(n)+\tilde{w}(n)
$$

where $\bar{h}(n)$ is the inverse system of $h(n)$, and $h(n) \otimes \bar{h}(n)=\delta(n)$,

$\delta(n)=\left\{\begin{array}{lc}1, & n=0 \\ 0, & \text { otherwise }\end{array}\right.$.

If ignoring the effects of AWGN, the output of circulated match-filtering becomes

$$
z(d)=\sum_{n=0}^{Q-1} g(n) p^{*}\left(\left(n-d N_{F T N}\right)\right)_{Q}=\sum_{n=0}^{Q-1} \sum_{d^{\prime}=0}^{D-1} a\left(d^{\prime}\right) p\left(\left(n-d^{\prime} N_{F T N}\right)\right)_{Q} p^{*}\left(\left(n-d N_{F T N}\right)\right)_{Q}
$$

Let

$$
c(d)=\sum_{n=0}^{L-1} p(n) p^{*}\left(\left(n-d N_{F T N}\right)\right)_{Q}
$$

then $\sum_{n=0}^{Q-1} p\left(\left(n-d^{\prime} N_{F T N}\right)\right)_{Q} p^{*}\left(\left(n-d N_{F T N}\right)\right)_{Q}=c\left(\left(d-d^{\prime}\right)\right)_{D}$. Thus, we have

$$
z(d)=\sum_{d^{\prime}=0}^{D-1} a\left(d^{\prime}\right) c\left(\left(d^{\prime}-d\right)\right)_{D}=a(d) \otimes c(d), \quad 0 \leq d \leq D-1
$$

Hence, $c(d)$ can be viewed as the equivalent impulse response of the ISI caused by FTN time squeezing. Since $p(n)$ is known at receiver, $c(d)$ can be calculated beforehand. Thus by FDE, the transmitted signal could be estimated as

$$
\hat{\mathbf{a}}=\mathbf{F}_{D}^{H} \boldsymbol{\Sigma} \mathbf{F}_{D} \mathbf{z}
$$

where $\hat{\mathbf{a}}$ and $\mathbf{z}$ are the vector expression of $\hat{a}(d)$ and $z(d)$ respectively, $\hat{a}(d)$ is the estimated value of $a(d) . \mathbf{F}_{D}$ and $\mathbf{F}_{D}^{H}$ are the $D \times D$ Fourier transform and inverse Fourier transform matrix, and $\mathbf{F}_{D}^{H} \mathbf{F}_{D}=\mathbf{I}_{D} . \boldsymbol{\Sigma}$ is the 
equalization matrix.

\section{Post-Processing SNR Analysis in AWGN Channel}

The vector form of the transmitted CB-FTN signal without the CP can be described as

$$
\mathbf{S}=\mathbf{P a}
$$

where $\mathbf{s}=\left[\begin{array}{llll}s(0) & s(1) & \cdots & s(Q-1)\end{array}\right]^{T}, \mathbf{a}=\left[\begin{array}{llll}a(0) & a(1) & \cdots & a(D-1)\end{array}\right]^{T}$, $\mathbf{P}=\left[\begin{array}{lllll}\mathbf{p}_{0} & \cdots & \mathbf{p}_{d} & \cdots & \mathbf{p}_{D-1}\end{array}\right], \quad \mathbf{p}_{0}=\left[\begin{array}{llll}p(0) & \cdots & p(L-1) & \mathbf{0}_{1 \times(Q-L)}\end{array}\right]^{T}$, and $\mathbf{p}_{d}$ denotes a vector obtained by cyclic down shifting $d \times N_{F T N}$ row of vector $\mathbf{p}_{0}$.

At the receiver, after CP removing and Q-tone channel FDE, the output signal can be given as

$$
\mathbf{r}=\mathbf{F}_{Q}^{H} \mathbf{H}^{H} \mathbf{W H} \mathbf{F}_{Q} \mathbf{P a}+\mathbf{F}_{Q}^{H} \mathbf{H}^{H} \mathbf{W} \mathbf{F}_{Q} \boldsymbol{w}
$$

where $w$ is the AWGN vector. Since the channel frequency response matrix $\mathbf{H}=\mathbf{I}_{Q}$ for AWGN channel, the equalization matrix for channel FDE can be designed as $\mathbf{W}=\mathbf{I}_{Q}$. As a result, the received signal can be reduced as

$$
\mathbf{r}=\mathbf{P a}+\mathbf{w}
$$

After circulated match-filtering, the output signal becomes

$$
\mathbf{z}=\mathbf{P}^{H} \mathbf{r}=\mathbf{P}^{H} \mathbf{P a}+\mathbf{P}^{H} \boldsymbol{w}
$$

According to (9), $\mathbf{P}^{H} \mathbf{P}$ is a $D \times D$ circulated symmetric matrix, and

$$
\mathbf{P}^{H} \mathbf{P}=\left(\begin{array}{cccc}
c(0) & c(1) & \cdots & c(D-1) \\
c(1) & c(0) & \cdots & c(D-2) \\
\vdots & \vdots & \cdots & \vdots \\
c(D-1) & c(D-2) & \cdots & c(0)
\end{array}\right)
$$

Therefore, the first term of (15) can be viewed as the output of vector a circulated convoluting with vector $\mathbf{c}$, and $\mathbf{c}=\left[\begin{array}{lllll}c(0) & c(1) & \cdots & c(D-1)\end{array}\right]^{T}$.

If the FDE utilized to cancel the ISI, the detect metric vector of demodulated symbols can be expressed as

$$
\hat{\mathbf{a}}=\mathbf{F}_{D}^{H} \mathbf{C}^{H} \boldsymbol{\Lambda} \mathbf{F}_{D} \mathbf{P}^{H} \mathbf{r}=\mathbf{F}_{D}^{H} \mathbf{C}^{H} \boldsymbol{\Lambda} \mathbf{C} \mathbf{F}_{D} \mathbf{a}+\mathbf{F}_{D}^{H} \mathbf{C}^{H} \boldsymbol{\Lambda} \mathbf{F}_{D} \mathbf{P}^{H} \boldsymbol{w}
$$

where $\mathbf{\Lambda}$ is the diagonal FDE matrix. $\mathbf{C}=\operatorname{diag}\{C(0) C(1) \cdots C(D-1)\}$, and

$$
\left[\begin{array}{llll}
C(0) & C(1) & \cdots & C(D-1)
\end{array}\right]^{T}=\sqrt{D} \mathbf{F}_{D}\left[\begin{array}{cccc}
C(0) & c(1) & \cdots & c(D-1)
\end{array}\right]^{T}
$$

For ZF FDE, the ISI equalization matrix is $\boldsymbol{\Lambda}=\operatorname{diag}\left\{1 / C(0)^{2} \quad 1 / C(1)^{2} \quad \cdots \quad 1 / C(D-1)^{2}\right\}$, then, $\mathbf{C}^{H} \mathbf{\Lambda} \mathbf{C}=\mathbf{I}_{D}$. As a result, the signal components, i.e. the first term in (17), can be given as

$$
\tilde{\mathbf{a}}=\mathbf{a}
$$

Assume $a(d)$ is the i.i.d. (independent and identically distributed) complex constellation symbols with zero mean and unit energy, the average energy of the signal in (19) can be given by

$$
E_{S}=E\left[|a(d)|^{2}\right]=1
$$


The noise components, i.e. the second term in (17) can be written as

$$
\tilde{\boldsymbol{w}}=\mathbf{F}_{D}^{H} \mathbf{C}^{H} \boldsymbol{\Lambda} \mathbf{F}_{D} \mathbf{P}^{H} \boldsymbol{w}
$$

The covariance matrix of noise vector can be expressed as

$$
\boldsymbol{\eta}=E\left[\tilde{\boldsymbol{w}} \tilde{\boldsymbol{w}}^{H}\right]=E\left[\mathbf{F}_{D}^{H} \mathbf{C}^{H} \boldsymbol{\Lambda} \mathbf{F}_{D} \mathbf{P}^{H} \boldsymbol{w} \boldsymbol{w}^{H} \mathbf{P} \mathbf{F}_{D}^{H} \boldsymbol{\Lambda}^{H} \mathbf{C} \mathbf{F}_{D}\right]=\sigma^{2} \mathbf{F}_{D}^{H} \boldsymbol{\Lambda}^{H} \mathbf{C F}_{D}
$$

Let

$$
\mathbf{U}=\boldsymbol{\Lambda}^{H} \mathbf{C}
$$

and

$$
\mathbf{U}=\operatorname{diag}\{1 / C(0) \quad 1 / C(1) \quad \cdots \quad 1 / C(D-1)\}
$$

Since $\mathbf{U}$ is a diagonal matrix, then $\mathbf{F}_{D}^{H} \mathbf{U F}_{D}$ is a $D \times D$ circulated symmetric matrix. Hence, the covariance matrix of noise becomes as

$$
\boldsymbol{\eta}=\sigma^{2} \mathbf{F}_{D}^{H} \mathbf{U F}_{D}=\sigma^{2}\left(\begin{array}{cccc}
u_{0} & u_{1} & \cdots & u_{D-1} \\
u_{1} & u_{0} & \cdots & u_{D-2} \\
\vdots & \vdots & \cdots & \vdots \\
u_{D-1} & u_{D-2} & \cdots & u_{0}
\end{array}\right)
$$

where

$$
\left[\begin{array}{llll}
u_{0} & u_{1} & \cdots & u_{D-1}
\end{array}\right]^{T}=\frac{1}{\sqrt{D}} \mathbf{F}_{D}^{H}\left[\begin{array}{llll}
U_{0} & U_{1} & \cdots & U_{D-1}
\end{array}\right]^{T}
$$

Therefore, we have $E\left[\tilde{w}_{d} \tilde{w}_{d}{ }^{H}\right]=u_{0}$, for $0 \leq d \leq D-1$, where $\tilde{\boldsymbol{w}}=\left[\begin{array}{llll}\tilde{w}_{0} & \tilde{w}_{1} & \cdots & \tilde{w}_{D-1}\end{array}\right]^{T}$.

Then by (26), the variance of the noise can be given by

$$
\sigma_{n}^{2}=\sigma^{2} u_{0}=\sigma^{2} \frac{1}{D} \sum_{d=0}^{D-1} U_{d}
$$

As a result, by (20) and (25), the post-processing SNR can be described as

$$
S N R=\frac{E_{S}}{\sigma_{n}^{2}}=1 /\left(\sigma^{2} \frac{1}{D} \sum_{d=0}^{D-1} 1 / C(d)\right)
$$

In fact, for Nyquist transmission, $N_{F T N}$ in (9) equals to $N$. According to the N-orthogonal property of $p(n), c(0)=1$ and $c(d)=0$, for $d \neq 0$. Therefore, by (18), $\quad \mathbf{C}=\mathbf{I}_{D}$. Then the post-processing SNR is reduced as

$$
S N R=1 / \sigma^{2}
$$

\section{Performance Evaluation of CB-FTN}

The performances of the proposed scheme are evaluated in this section. The system parameters for simulations are presented in Table 1.

\subsection{The Effects of the ISI Caused by FTN Time Squeezing}

In order to achieve higher spectrum efficiency, the CB-FTN system stuffs more data symbols in one data block than Nyquist system, which will cause the ISI within one block inevitably. Due to the cyclic property of CB-FTN signaling, according to (9), the equivalent impulse response of the ISI caused by FTN time 
Table 1. System parameters.

\begin{tabular}{ccc}
\hline & \multicolumn{2}{c}{ Simulation Systems } \\
\cline { 2 - 3 } Parameters & $\begin{array}{c}\text { Nyquist rate } \\
\text { transmission }\end{array}$ & $\begin{array}{c}\text { FTN rate } \\
\text { transmission }\end{array}$ \\
\hline Modulation scheme & \multicolumn{2}{c}{ QPSK } \\
Rype of pulse-shaping filter & RRC \\
Roll-off factor & 0.3 \\
Length of filter $(L)$ & 241 \\
Up-sampling rate $\left(N_{S}\right)$ & 20 \\
\# of shift samples of shaping filtering $\left(N_{F T N}\right)$ & 20 & 16,18 \\
\# of symbols carried by one circulated block $(\mathrm{D})$ & 16 & 20 \\
Length of circulated block $(\mathrm{Q})$ & 320 & 320,360 \\
Time squeezing ratio $(\xi)$ & 1 & $0.8,0.9$ \\
Spectrum efficiency $(\mathrm{bps} / \mathrm{Hz})$ & 2 & $2.5,2.22$ \\
\hline
\end{tabular}

squeezing is symmetric, as shown in Figure 3(a). We also notice that $c(d)$ trends to $\delta(n)$, when $N_{F T N}$ increases to $N_{S}$, i.e. $\xi$ to 1 . As shown in Figure 3(b), frequency selectivity of $c(d)$ becomes severe with the decrease of $N_{F T N}$, i.e. the high frequency components of transmitted signal is suppressed.

\subsection{Post-Processing SNR with ZF-FDE for ISI Cancelation}

Figure 4 presents the both theoretical and simulated post-processing SNR of the CB-FTN receiver with zero-forcing FDE for ISI cancelation in AWGN channel. As shown in the Figure 4(a), the post-processing SNR is increased with $\xi$. Due to the severe frequency selectivity, the post-processing SNR is much lower than the received SNR when $\xi=0.8$, i.e. $N_{F T N}=16$. Meanwhile, for Nyquist rate transmission, i.e. $\xi=1$ or $N_{F T N}=20$, the post-processing SNR is equal to the received SNR. Furthermore, within the received SNR range, the theoretical results are well matched with the simulation results.

In order to implement FDE with fast Fourier transform, the length of circulated block $Q$ should be chosen as a power of two. For example, if set $N_{F T N}=16$ and $D=32$, we can get $Q=512$. When the up-sampling rate of pulse-shaping is set $N=20$, it can be obtained that the time squeezing ratio $\xi=0.8$. Figure 4(b) compares the post-processing SNRs of the CB-FTN system with different sample block length. As shown in the figure, with the same time squeezing ratio $\xi$, the systems with different $D$ achieve the similar performances. With the increasing of $D$, the post-processing SNR is increased slightly, because the frequency selectivity of $c(d)$ is reduced a little with the increase of $D$.

The BER performances of CB-FTN system with turbo coding and ZF-FDE are illustrated in Figure 5. By comparing with Figure 4(a), it can be noticed that the effects of time squeezing ratio on the BER performance are similar to that on post-processing SNR. Moreover, BER performances of CB-FTN system are almost the same as that of the scheme proposed in [9], that is because the principle 


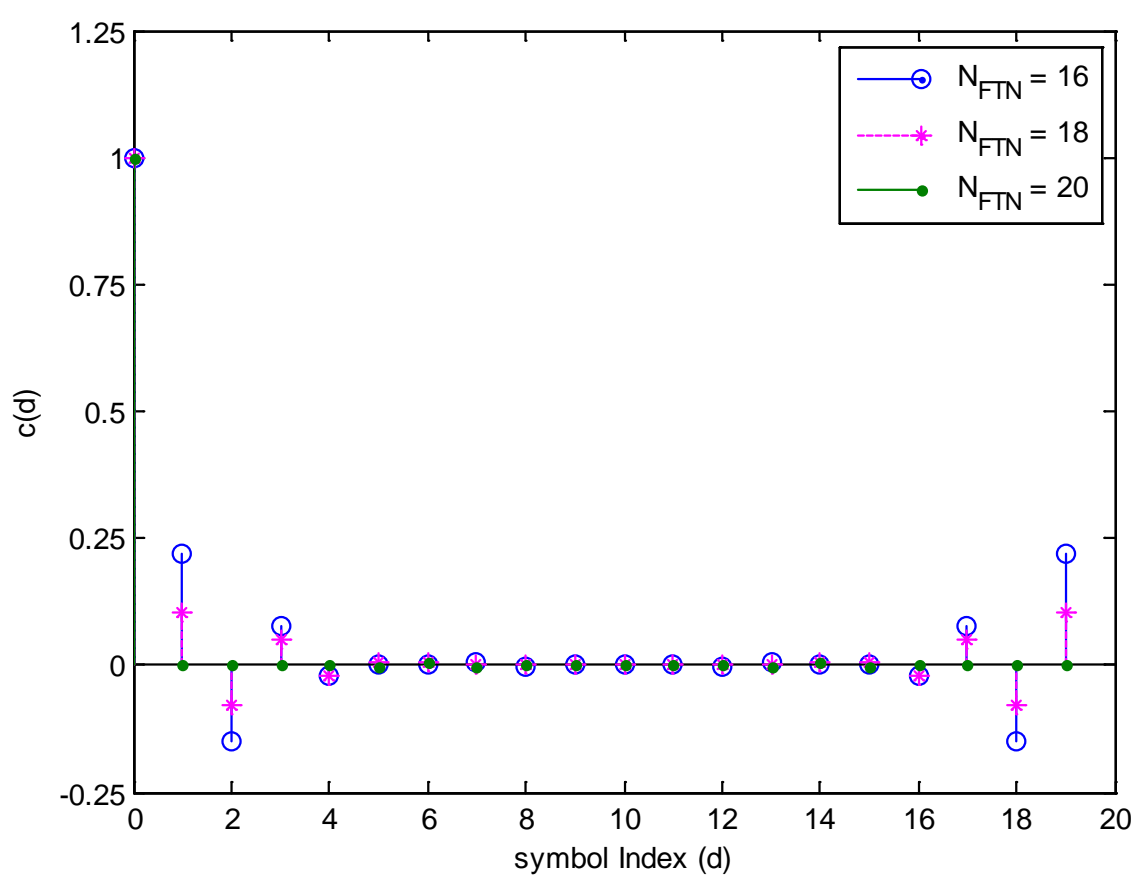

(a)

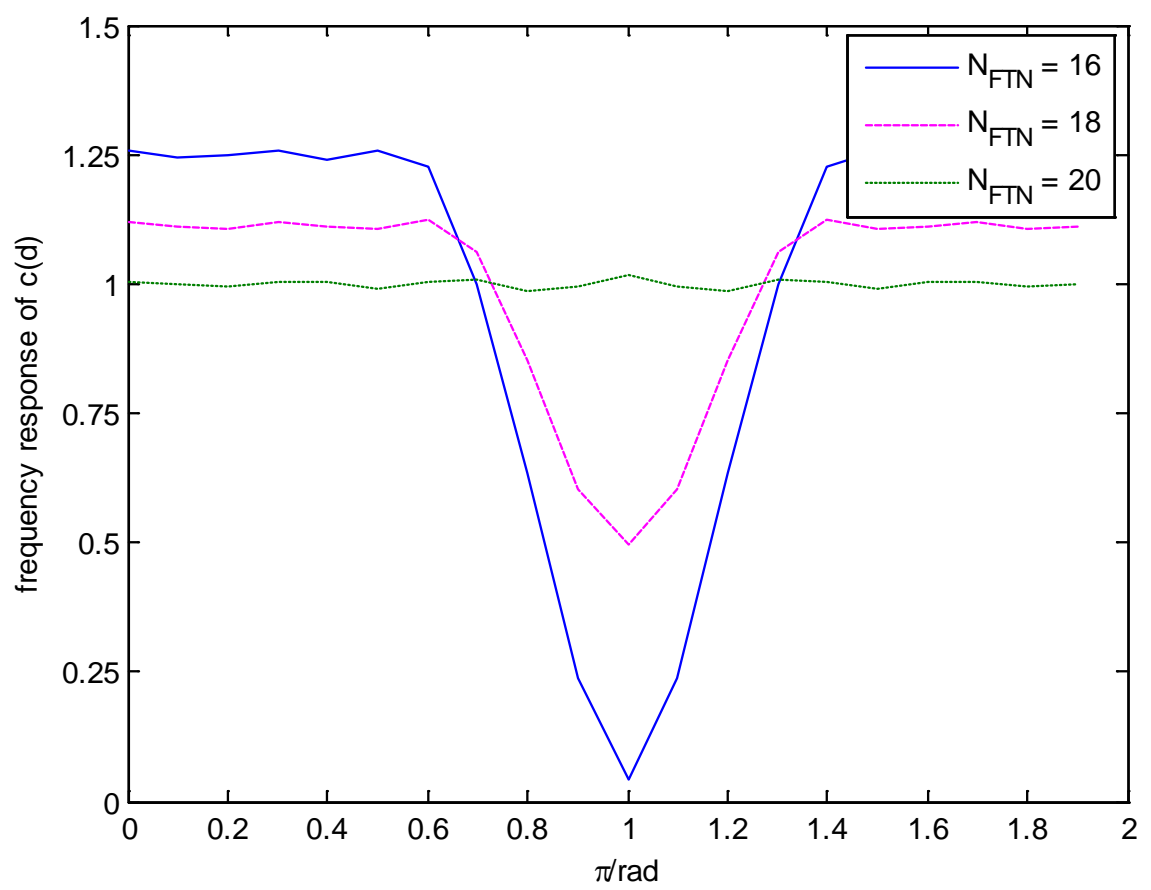

(b)

Figure 3. Time-/frequency domain property of $c(d)$.

of transmitters of both schemes are similar, but with different implementation structure.

As mentioned in above section, the scheme in [9] needs symbol-based CP/CS inserting and sample-based CP/CS removing operations before and after pulseshaping filtering respectively, which will increase the computational complexity of transmitter. For example, if set $L=241, N_{F T N}=16$, and $D=32$, i.e. $Q=$ 


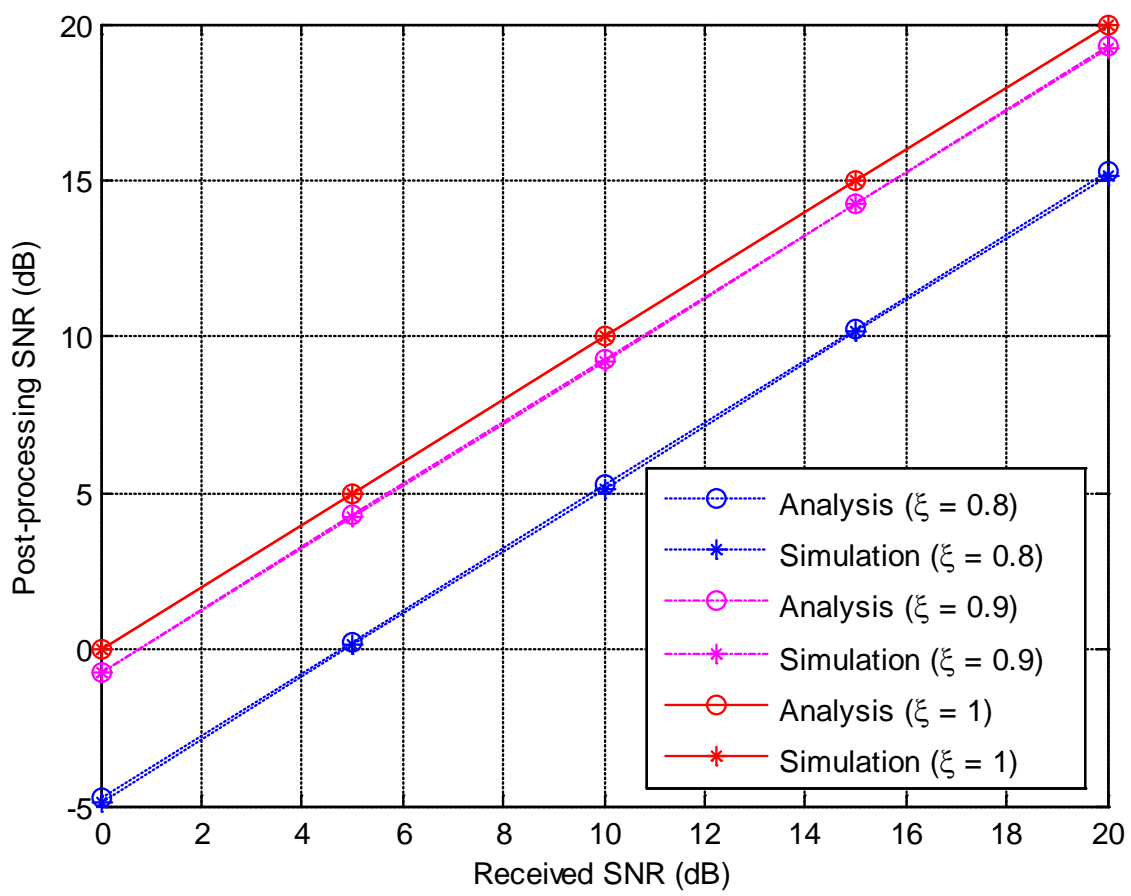

(a)

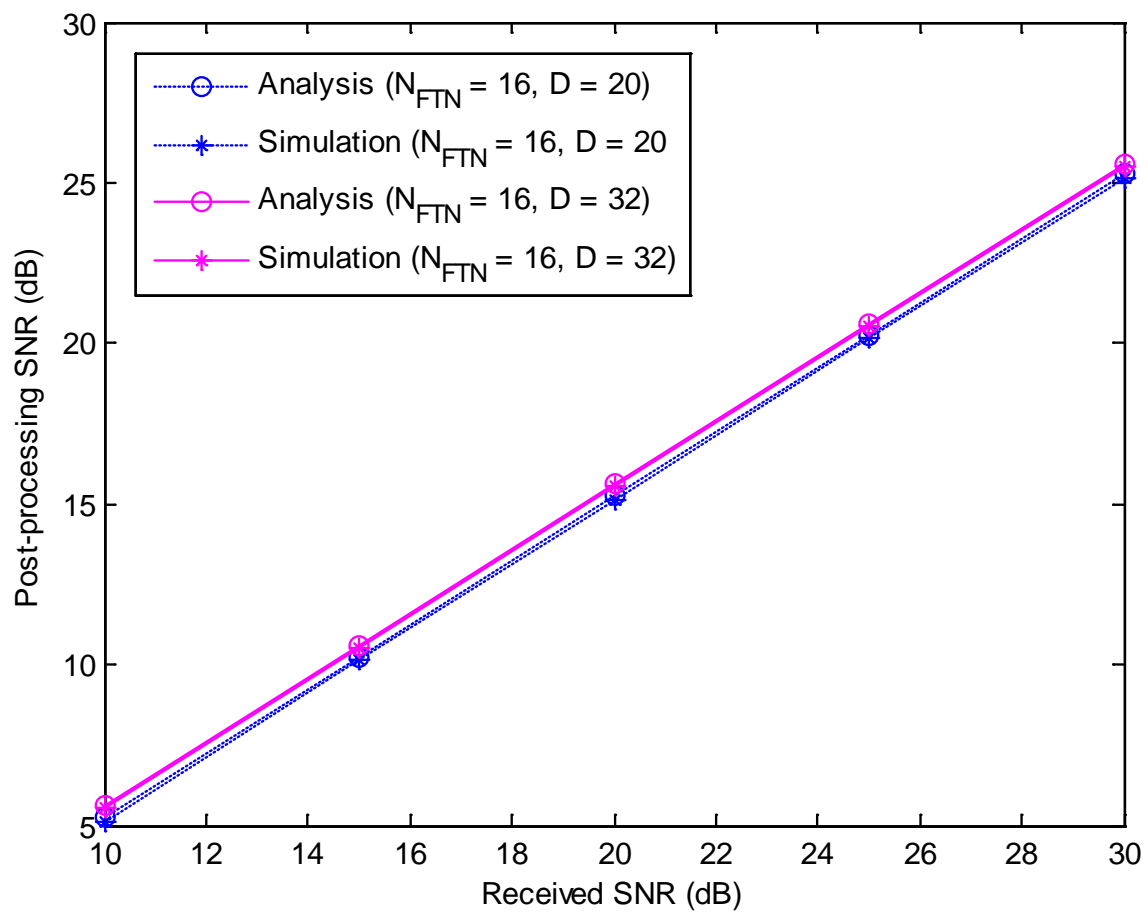

(b)

Figure 4. Post-processing SNR with ZF-FDE. (a) With different $\xi$. (b) With different $D$.

512, the length of symbol-based CP/CS should be $P=\left\lfloor(L / 2) / N_{F T N}\right\rfloor=8$, so as to form the circulated sample block. Consequently, an additional $50 \%$ of computational complexity is needed, since the CP/CS with total length 16 , which will be discarded before final transmission, should be passed through the pulseshaping filter for transmission of 32 data symbols. However, the above redundant 


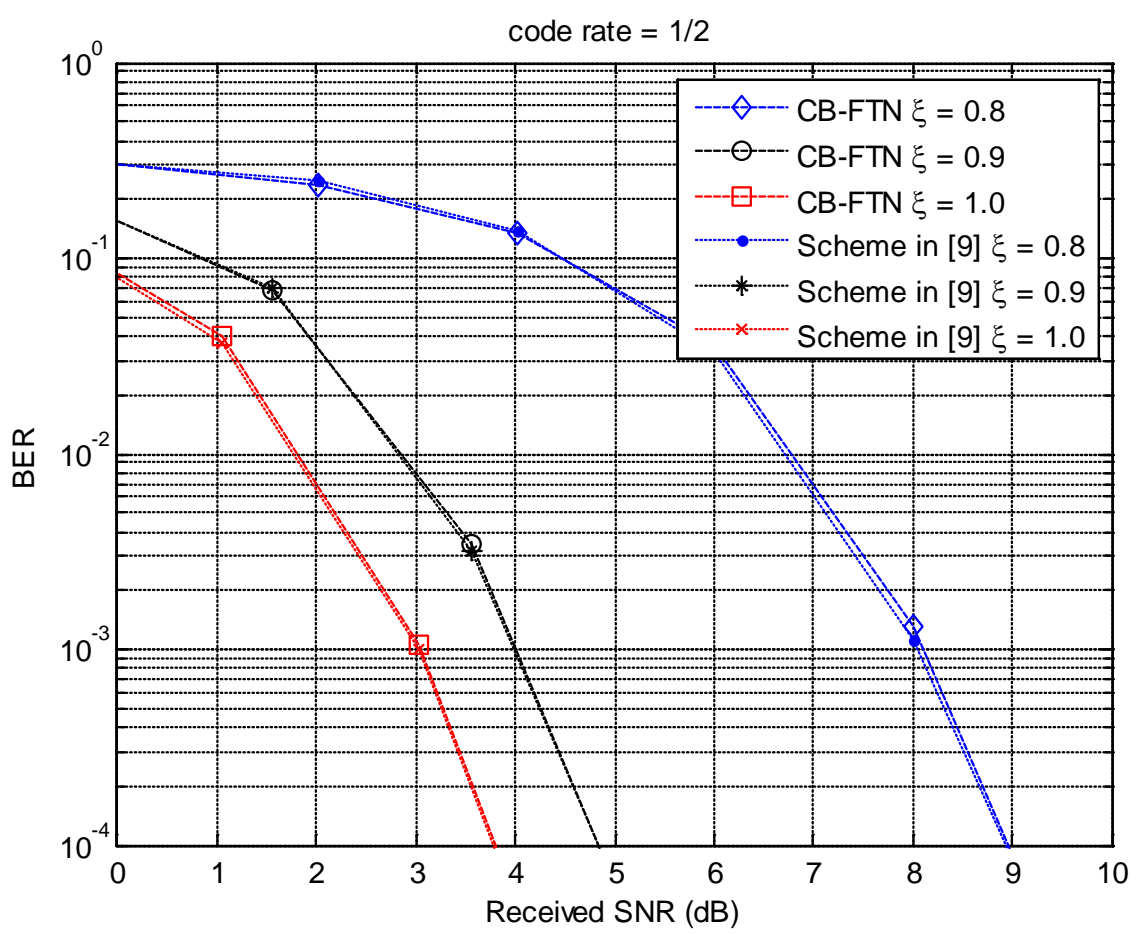

Figure 5. BER performance in AWGN channel.

operations are unnecessary for the CB-FTN transmitter utilizing circulated blocking operation to form the circulated sample block, which is equivalent to only 32 data symbols passed through the pulse-shaping filter.

\section{Conclusion}

In this paper, a circulated block transmission scheme is proposed for FTN signaling, i.e. CB-FTN system. By circulated sample blocking and CP padding, the FDE can be utilized to cancel the IBI induced by wireless multipath channel. Moreover, by exploiting the circulation property of the sample block, the ISI caused by FTN filtering, can be eliminated by FDE with the circular self-correlation function of pulse-shaping filter as well. The equivalent impulse response and effects of the ISI are illustrated and analyzed by simulation. The theoretical post-processing SNR are analyzed for the CB-FTN receiver with ZF FDE in AWGN channel, which is verified by the simulation results. Moreover, the BER performances of the CB-FTN system are illustrated and compared with the existed scheme. It can be found that both schemes have very close BER performances, but the former has much lower computational complexity of transmitter than the latter.

\section{Acknowledgements}

This work is supported by the International cooperation project of National Natural Science Foundation of China (No. 6146136001), and the capability improvement project of Zhangjiang Administrative Committee of Shanghai Municipality (No. 2016-14). 


\section{References}

[1] Mazo, J. (1975) Faster-than-Nyquist Signaling. The Bell System Technical Journal, 54, 1451-1462. https://doi.org/10.1002/j.1538-7305.1975.tb02043.x

[2] Liveris, A.D. and Georghiades, C.N. (2003) Exploiting Faster-than-Nyquist Signaling. IEEE Transactions on Communications, 51, 1502-1511. https://doi.org/10.1109/TCOMM.2003.816943

[3] McGuire, M. and Sima, M. (2010) Discrete Time Faster-than-Nyquist Signaling. Proc. IEEE Global Telecommun. Conf., Miami, FL, Dec. 2010, 1-5.

[4] Prlja, A. and Anderson, J.B. (2012) Reduced-Complexity Receivers for Strongly Narrowband Intersymbol Interference Introduced by Faster-than-Nyquist Signaling. IEEE Transactions on Communications, 60, 2591-2601. https://doi.org/10.1109/TCOMM.2012.070912.110296

[5] Nie, S., Guo, M. and Shen, Y. (2015) A New Equalization Scheme for Faster-than-Nyquist Signaling. IEEE 6 th International Conference on Wireless, Mobile and Multi-Media (ICWMMN2015), Beijing, China, Nov. 2015, 74-77.

[6] Sugirua, S. and Hanzo, L. (2015) Frequency-Domain Equalization Aided Iterative Detection of Faster-than-Nyquist Signaling. IEEE Transactions on Vehicular Technology, 64, 2122-2128. https://doi.org/10.1109/TVT.2014.2336984

[7] Yuan, W.J., Wu, N., Wang, H. and Kuang, J.M. (2016) Variational Inference-Based Frequency-Domain Equalization for Faster-than-Nyquist Signaling in Doubly Selective Channels. IEEE Signal Processing Letters, 23, 1270-1274. https://doi.org/10.1109/LSP.2016.2593058

[8] Sugiura, S. (2013) Frequency-Domain Equalization of Faster-than-Nyquist Signaling. IEEE Wireless Communications Letters, 2, 555-558. https://doi.org/10.1109/WCL.2013.072313.130408

[9] Hong, S.B. and Seo, J.S. (2016) Cyclic Prefix/Suffix-Assisted Frequency-Domain Equalization for Faster-than-Nyquist Signaling Block Transmission. IEEE 27 th Annual IEEE International Symposium on Personal, Indoor and Mobile Radio Communications, Valencia, Spain, 4-8 September 2016. https://doi.org/10.1109/PIMRC.2016.7794784

[10] Zhang, X.D., Li, M.Q., Hu, H.L., et al. (2006) DFT Spread Generalized Multi-Carrier Scheme For Broadband Mobile Communications. IEEE International Symposium on Personal, Indoor and Mobile Radio Communications, Helsinki, Finland, 11-14 September 2006, 1-5. https://doi.org/10.1109/PIMRC.2006.254321

[11] Li, M. and Zhang, X. (2009) Performance Analysis of DFT Spread Generalized Multi-Carrier Systems. Science in China Series F: Information Sciences, 52, 2385-2396. https://doi.org/10.1007/s11432-009-0199-1 
Submit or recommend next manuscript to SCIRP and we will provide best service for you:

Accepting pre-submission inquiries through Email, Facebook, LinkedIn, Twitter, etc. A wide selection of journals (inclusive of 9 subjects, more than 200 journals)

Providing 24-hour high-quality service

User-friendly online submission system

Fair and swift peer-review system

Efficient typesetting and proofreading procedure

Display of the result of downloads and visits, as well as the number of cited articles Maximum dissemination of your research work

Submit your manuscript at: http://papersubmission.scirp.org/

Or contact ijcns@scirp.org 